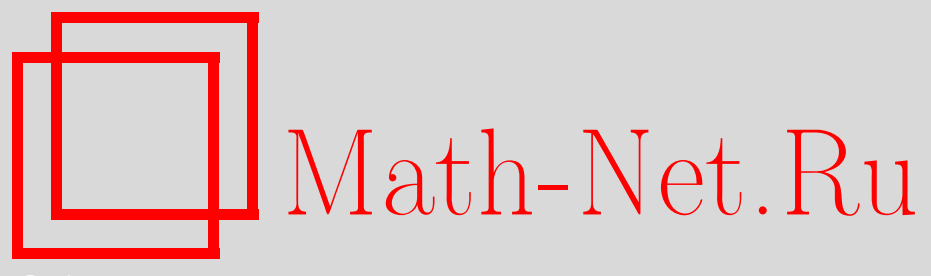

А. Т. Ильичев, В. В. Козлов, Д. В. Трещёв, А. П. Чугайнова, Андрей Геннадьевич Куликовский (к восьмидесятилетию со дня рождения), УМН, 2013, том 68, выпуск $6,179-188$

DOI: https://doi.org/10.4213/rm9562

Использование Общероссийского математического портала Math-Net.Ru подразумевает, что вы прочитали и согласны с пользовательским соглашением http://www . mathnet.ru/rus/agreement

Параметры загрузки:

IP : 54.80 .73 .141

26 апреля 2023 г., 17:54:37

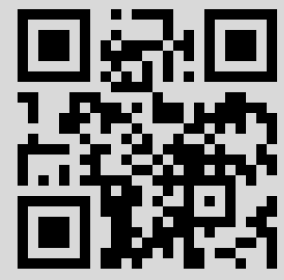




\section{МАТЕМАТИЧЕСКАЯ ЖКИЗЬЬ}

\section{Андрей Геннадьевич Куликовский} (к восьмидесятилетию со дня рождения)

18 марта 2013 г. - день восьмидесятилетия действительного члена Российской академии наук Андрея Геннадьевича Куликовского.

А. Г. Куликовский родился в Москве. В 1950 г. он поступил на механико-математический факультет Московского государственного университета на отделение механики, где еще в студенческие годы проявились его незаурядные научные способности и присущий ему в высшей степени глубокий аналитически-инженерный склад ума. При распределении по специальностям тогдашний декан мехмата (а впоследствии академик, выдающийся отечественный ученый в области упругости и пластичности) Ю.Н. Работнов направил его в группу вычислителей. Однако А. Г. Куликовского привлекала гидромеханика и он, заручившись поддержкой Л. И. Седова, поступил на кафедру гидромеханики. Л. И. Седов стал заботливым и внимательным учителем

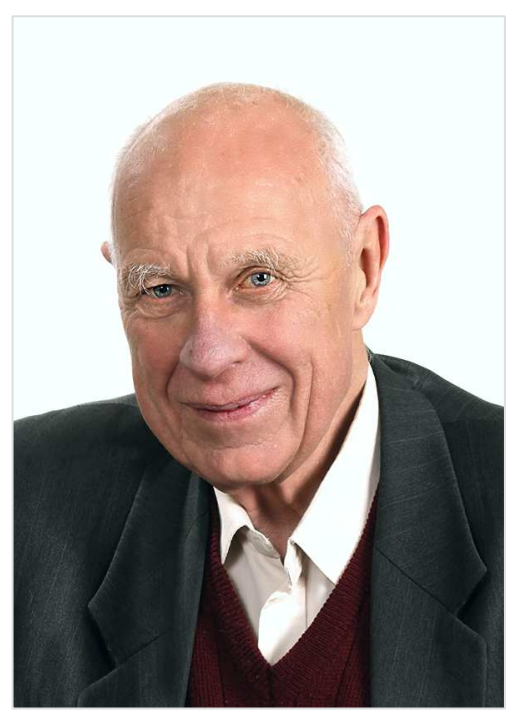
А.Г. Куликовского, и начало самостоятельного пути его в науке связано с именем этого великого ученого.

Закончив университет в 1955 г., А. Г. Куликовский поступил в аспирантуру на кафедру гидромеханики к Л.И. Седову, в 1958 г. он защитил кандидатскую диссертацию, после чего долгие годы работал в Математическом институте им. В. А. Стеклова: с 1959 по 1988 г. младшим, старшим и ведущим научным сотрудником, с 1988 по 2003 г. как заведующий отделом механики, а с 2003 г. как главный научный сотрудник института. За это время А. Г. Куликовский добился значительных успехов, которые были высоко оценены коллегами: в 1969 г. он защитил докторскую диссертацию, в 1991 г. был избран членом-корреспондентом, а в 2006 г. - действительным членом (академиком) РАН по Отделению энергетики, машиностроения, механики и процессов управления.А.Г.Куликовский - блестящий представитель школы Л. И. Седова и всей отечественной науки, ее гордость и по праву считается одним из немногих ведущих специалистов и уникальным экспертом по механике сплошной среды в нашей стране. Он является лауреатом Государственной премии РФ, премии РАН им. С. А. Чаплыгина, премии и медали им. Л. И. Седова Российского национального комитета по теоретической и прикладной механике и главным редактором журнала "Известия Российской академии наук. Механика жидкости и газа". А. Г. Куликовский - автор и соавтор свыше 100 научных работ, в том числе шести монографий. В результате своей

DOI: $10.4213 / \mathrm{rm} 9562$ 
преподавательской и педагогической деятельности на механико-математическом факультете МГУ им. М. В. Ломоносова он воспитал 16 кандидатов и 9 докторов наук.

Первые важные результаты А.Г. Куликовского относятся к мало исследованной в то время области - магнитной гидродинамике, которой он стал заниматься по предложению академика Л. И. Седова, обладавшего ясным видением научной перспективы. А.Г. Куликовским исследованы нелинейные волны Римана и возникновение ударных волн в процессе их опрокидывания [9]; впервые исследована проблема обтекания тел, имеющих собственное магнитное поле, потоком идеально проводящей жидкости и построены примеры таких течений с образованием около тела каверны области, куда не проникает поток [8]. Совместно с Г.А. Любимовым им написана первая в мире монография по магнитной гидродинамике (1962 г.; в 2005 г. вышло дополненное издание [1]). А.Г. Куликовский рассмотрел течения слабо проводящей жидкости и построил общие решения при различном задании граничных условий и внешнего приложенного поля [23], [1].

Совместно с Г. А. Любимовым и А. А. Барминым А. Г. Куликовский создал теорию ударных волн и фронтов ионизации и рекомбинации в электромагнитном поле [10], [17], [18], [20], [22], [26], [37], [1], [3]. Эти фронты, через которые протекает газ, служат границей между электропроводным газом, подчиняющимся уравнениям магнитной гидродинамики, и областью, где газ неэлектропроводный и подчиняется уравнениям газовой динамики, а электромагнитное поле описывается уравнениями Максвелла. Им было выяснено, что эти фронты обладают необычными свойствами, не встречавшимися ранее. С помощью рассмотрения структуры разрывов было показано, что ударная адиабата, т. е. множество состояний за фронтом, распространяющимся по заданному состоянию, может содержать одно-, двух- и трехмерные части. В этих случаях из требования существования структуры разрыва были получены дополнительные соотношения, которые, наряду с основными соотношениями, следующими из законов сохранения, должны выполняться на разрыве. Число дополнительных соотношений равнялось соответственно двум, единице и нулю. Были рассмотрены тестовые автомодельные задачи, содержащие такие разрывы.

Как обобщение результатов, полученных при изучении фронтов ионизации и рекомбинации, А.Г. Куликовский доказал следующее утверждение. Если число основных соотношений на некотором разрыве в решениях гиперболических уравнений (по разные стороны разрыва уравнения могут быть различными) меньше, чем требуется условиями корректности граничных условий на разрыве, то требование существования решения о структуре этого разрыва (если структура существует) приводит к необходимости выполнения как раз стольких дополнительных соотношений на разрыве (граничных условий), сколько требуется для корректности (см. [18], [1], [2], [3]).

Совместно с Е.И. Свешниковой проведено исследование нелинейных квазипоперечных волн малой амплитуды в слабоанизотропных упругих средах [2], [31], [34], [38], [40], [41], [42], [46], [48], [55]. В предположении, что амплитуда волн мала (в уравнениях учитывались члены третьей степени по амплитуде), было обнаружено сложное поведение волн, которое удалось полностью исследовать аналитически. Были решены важнейшие тестовые автомодельные классические задачи в предположении, что ударные волны априорно эволюционны (т. е. условия на них корректны в силу законов сохранения). Было проведено исследование структуры разрывов с учетом вязкости и показано, что априорно-эволюционные волны имеют структуру. Однако решения автомодельных задач в указанной постановке в некоторой области параметров оказались двузначными. Вопрос о том, какое из этих решений осуществляется, был решен А. П. Чугайновой, ученицей А. Г. Куликовского. Была численно рассмотрена неавтомодельная задача, решение которой, как можно было ожидать, имеет при больших временах автомодельную асимптотику. Обнаружено, что решение действительно выходит на ту или другую асимптотику, но на какую именно, зависит от деталей не 
автомодельной постановки задачи (эти детали отсутствуют в автомодельной постановке). Изложение этих и других результатов и соответствующие ссылки представлены в [5], [61], [3]. Было показано, что все априорно эволюционные разрывы устойчивы по отношению как к одномерным, так и к двумерным возмущениям [52], [53], [56].

В рамках исследования квазипоперечных одномерных волн была исследована, совместно с Е. И. Свешниковой, задача о резонансе колебаний в слое анизотропной упругой среды при наличии малой периодической возбуждающей силы на одной из границ. Были получены уравнения, описывающие зависимость решения на одной из границ от времени, и исследованы условия и процесс возникновения разрывов [65].

Большой цикл работ, касающихся влияния мелкомасштабной дисперсии, которая появляется в структуре разрывов, был начат работой А. Г. Куликовского [33], в которой рассмотрено модельное уравнение типа Кортевега-де Фриза-Бюргерса, но с более сложной нелинейностью, такой же, как в известной работе О. А. Олейник. Было выяснено, что, наряду с ударными волнами, как результат влияния дисперсии появляется множество новых, особых разрывов с дополнительным соотношением, следующим из требования существования решения о структуре. Типы разрывов различаются тем, что внутри структуры за счет дисперсии может происходить одно, два и более колебаний решения. Максимальное число таких колебаний и, соответственно, типов особых разрывов зависит от соотношения между дисперсией и диссипацией в структуре разрыва. При этом у тестовых автомодельных задач может оказаться много решений.

Далее это направление развивалось в совместных работах с Н. И. Гвоздовской (см., например, [51]), ученицей А. Г. Куликовского, в которых рассматривались нелинейные волны в магнетиках (где дисперсия обеспечивается прецессией спинов электронов), а также в совместных работах с А.П. Чугайновой (и в ее собственных работах), в которых рассматривались нелинейные квазипоперечные волны в упругих средах при наличии дисперсии (в упругих телах дисперсия волн возникает при наличии микроструктуры, как в случае композитных сред) [3], [5], [61]-[63]. В последнем случае помимо автомодельных задач исследовались неавтомодельные задачи, связанные с выходом решений на одну из автомодельных асимптотик, которых может быть много при преобладающей дисперсии в структуре разрывов. Были выявлены закономерности реализации тех или иных автомодельных асимптотик как в случае упругих сред с дисперсией, так и в случае упомянутого выше модельного уравнения. Реализация той или иной асимптотики зависит от деталей неавтомодельной постановки задачи, отсутствующих в условиях автомодельной задачи. Эти результаты изложены в [3], [6], [63].

Другой круг задач, который всегда привлекал А. Г. Куликовского, - это проблемы устойчивости. Серия работ, совместных с Ф.А. Слободкиной, посвящена изучению устойчивости и развития возмущений в одномерных течениях в окрестности точки изменения знака одной из характеристических скоростей (точки "перехода через скорость звука") [15], [32]. Найден критерий роста нелинейных возмущений. Результаты применены к магнитогидродинамическим течениям.

Большой интерес представляет задача выяснения характера неустойчивости однородных (не зависящих от координат) течений и состояний во всем пространстве. Был известен критерий, позволяющий различить абсолютную неустойчивость, когда первоначально локализованное возмущение растет со временем во всех точках пространства, и конвективную неустойчивость, когда такое возмущение растет, но перемещается и с течением времени уходит из любой точки. А. Г. Куликовский показал, что наличие удаленных граничных условий может дестабилизировать ситуацию: во-первых, в случае, когда сами граничные условия совместно с уравнениями служат источниками растущих возмущений (граничная неустойчивость), и, во-вторых, в случае, когда образуется самоподдерживающаяся растущая со временем замкнутая цепочка волн, 
превращающихся одна в другую в результате взаимодействия с разными границами (глобальная неустойчивость). В том и другом случае, когда точки, в которых выставлены граничные условия, разнесены достаточно далеко, были получены асимптотические формы уравнений для нахождения собственных частот [14]. Результаты были обобщены на случай фона, медленно меняющегося в пространстве [35]. Идеи глобальной неустойчивости были применены В. В. Веденеевым, учеником А. Г. Куликовского, для исследования проблем возникновения флаттера пластин в сверхзвуковом потоке газа.

В совместных работах с И. С. Шикиной [36], [50] в приближении Рэлея была рассмотрена задача о развитии малых возмущений поверхности однородного неустойчивого тангенциального разрыва в идеальной жидкости в случае, когда начальные данные представляют собой $\delta$-функцию. Было показано, что при больших временах область, занятая экспоненциально растущими возмущениями, расширяется линейно со временем и при $t=$ const имеет форму, напоминающую разрезанное яблоко.

В последнее время А. Г. Куликовский совместно с Н. Т. Пащенко [60], [64], [67] получил ряд результатов о развитии во времени малых возмущений на слабо неоднородном фоне.

В области разрывных решений нелинейных гиперболических систем уравнений предметом изучения были ситуации, когда число законов сохранения меньше порядка системы уравнений [66]. Это имеет место, в частности, в теории упруго-пластичности. Образование разрывов при опрокидывании волн Римана в упруго-пластических средах изучено в совместной работе с А. П. Чугайновой [68].

Особо следует отметить действующий под руководством А. Г. Куликовского (совместно с В. П. Карликовым и О.Э. Мельником) семинар. Семинар является ведущим по механике сплошной среды в нашей стране, и возможность выступить на нем очень высоко ценится отечественными учеными. Одобрение результатов доклада на семинаре имеет определяющее значение при оценке их научного статуса, решении о публикации в центральных отечественных журналах, защите диссертаций и приглашении выступить с докладом на крупнейших отечественных конференциях и съездах по механике сплошных сред.

Несмотря на то что А.Г. Куликовский является принципиальным и даже порой жестким в оценке научных результатов, в повседневной жизни он душевный, доброжелательный, добросердечный и мягкий человек. И за это сочетание качеств (увы, редкое в наше время) его любят не только его друзья и многочисленные ученики, но также его коллеги и те, кто сколько-нибудь его знает.

Свой юбилей А. Г. Куликовский встречает в полном расцвете творческих сил, удивляя более молодых коллег своей работоспособностью и высоким качеством научных результатов. От всего сердца поздравляем юбиляра и желаем ему здоровья, счастья и новых ярких результатов.

А. Т. Ильичев, В.В. Козлов, Д. В. Трещёв, А. П. Чугайнова

\section{Список основных трудов А.Г. Куликовского}

\section{Монографии и лекционные курсы}

[1] Магнитная гидродинамика, Логос, М., 2005, 325 с. (совм. с Г. А. Любимовым).

[2] Нелинейные волны в упругих средах, Московский лицей, М., 1998, 412 с. (совм. с Е. И. Свешниковой).

[3] Математические вопросы численного решения гиперболических систем уравнений, изд. 2-е, испр. и доп., МАИК, Физматлит, М., 2012, 656 с. (совм. с Н. В. Погореловым, А. Ю. Семеновым). 
[4] Механика сплошных сред в задачах, т. 1, 2, ред. М.Э. Эглит, Московский лицей, М., 1996, 396 с., 394 с. (совм. с Г. Я. Галиным, А. Н. Голубятниковым, Я. А. Каменяржем, В.П. Карликовым, А. Г. Петровым, Е. И. Свешниковой, И. С. Шикиной, М.Э. Эглит); англ. пер.: Continuum mechanics via problems and exercises, v. 1, 2, World Sci. Ser. Nonlinear Sci. Ser. A Monogr. Treatises, 19, eds. M. E. Eglit, D. H. Hodges, World Scientific Publishing Co., Inc., River Edge, NJ, 1996, xiv+270 pp., xii+253 pp. (with M. E. Eglit, A. N. Golubiatnikov, J. A. Kamenjarzh, V.P. Karlikov, A. G. Petrov, I. S. Shikina, E. I. Sveshnikova).

[5] "Классические и неклассические разрывы и их структуры в нелинейно-упругих средах с дисперсией и диссипацией", Совр. пробл. матем., 7, МИАН, М., 2007, 3-148 (совм. с А.П. Чугайновой); англ. пер.: "Classical and nonclassical discontinuities and their structure in nonlinear elastic media with dispersion and dissipation", Proc. Steklov Inst. Math., 276:suppl. 2 (2012), 1-68 (with A. P. Chugainova).

[6] "Математические методы изучения разрывных решений нелинейных гиперболических систем уравнений”, Лекц. курсы НОЦ, 16, МИАН, М., 2010, 3-120 (совм. с Е. И. Свешниковой, А. П. Чугайновой).

\section{Основные научные статьи}

[7] "О вырождении однородной изотропной магнитогидродинамической турбулентности в несжимаемой жидкости”, ПММ, 19:5 (1955), 551-556.

[8] "Об обтекании намагниченных тел проводящей жидкостью”, Докл. АН CCCP, 117:2 (1957), 199-202.

[9] “О волнах Римана в магнитной гидродинамике”, Докл. АН СССР, 121:6 (1958), 987-990.

[10] "О магнитогидродинамических ударных волнах, ионизирующих газ", Докл. АH CCCP, 129:1 (1959), 52-55 (совм. с Г. А. Любимовым); англ. пер.: "Gas-ionizing magnetohydrodynamic shock waves", Soviet Phys. Dokl., 4 (1960), 1185-1188 (with G. A. Lyubimov).

[11] “О структуре ударных волн”, ПММ, 26:4 (1962), 631-641; англ. пер.: "Тhе structure of shock waves", J. Appl. Math. Mech., 26:4 (1962), 950-964.

[12] "Об общем условии устойчивости высших корреляционных функций в плазме", Докл. АН СССР, 156:1 (1964), 35-37 (совм. с С. В. Иорданским); англ. пер.: "A general stability condition for higher correlation functions in a plasma", Soviet Phys. Dokl., 9 (1964), 347-349 (with S. V. Iordanskii).

[13] "Об абсолютной устойчивости некоторых плоскопараллельных течений при больших числах Рейнольдса", ЖЖЭТФ, 49:5 (1965), 1326-1331 (совм. с С. В. Иорданским); англ. пер.: "The absolute stability of some plane parallel flows at high Reynolds numbers", Soviet Physics JETP, 22 (1966), 915-918 (with S. V. Iordanskii).

[14] "Об устойчивости однородных состояний”, ПММ, 30:1 (1966), 148-153; англ. пер.: "On the stability of homogeneous states", J. Appl. Math. Mech., 30:1 (1966), 180-187.

[15] "Об устойчивости произвольных стационарных течений в окрестности точек перехода через скорость звука”, ПММ, 31:4 (1967), 593-602 (совм. с Ф. А. Слободкиной); англ. пер.: "Equilibrium of arbitrary steady flows at the transonic points", J. Appl. Math. Mech., 31:4 (1967), 623-630 (with F. A. Slobodkina).

[16] "О медленных стационарных течениях проводящей жидкости при больших числах Гартмана", Изв. АН СССР. Сер. механ. жидкости и газа, 3:2 (1968), 3-10; англ. пер.: "Slow steady flows of a conducting fluid at large Hartmann numbers", Fluid Dynam., 3:2 (1968), 1-5.

[17] "Об ударных волнах, ионизующих газ, находящийся в электромагнитном поле", Докл. АН СССР, 178:1 (1968), 55-58 (совм. с А. А. Барминым); англ. пер.: "Shock 
waves of ionized gases in an electromagnetic field", Soviet Phys. Dokl., 13:1 (1968), 4-7 (with A. A. Barmin).

[18] "О поверхностях разрыва, разделяющих идеальные среды с различными свойствами. Волны рекомбинации в магнитной гидродинамике", ПМM, 32:6 (1968), 1125-1131; англ. пер.: "Surfaces of discontinuity separating two perfect media of different properties. Recombination waves in magnetohydrodynamics", J. Appl. Math. Mech., 32:6 (1968), 1145-1152.

[19] "Об устойчивости и эволюционности распределения электрического тока в среде с нелинейной проводимостью", ПММ, 32:4 (1968), 761-762 (совм. с С. А. Регирером); англ. пер.: "On stability and evolution of the electric current distribution in a medium with nonlinear conductivity", J. Appl. Math. Mech., 32:4 (1968), 792-794 (with S. A. Regirer).

[20] "Изменение скорости газа в ионизующих ударных волнах. Задача о проводящем поршне", ПММ, 32:3 (1968), 495-499 (совм. с А. А. Барминым); англ. пер.: "Gas velocity variation in ionizing shock waves. The problem of the conductive piston", J. Appl. Math. Mech., 32:3 (1968), 506-510 (with A. A. Barmin).

[21] "Модель взаимодействия солнечного ветра с межзвездной средой", Докл. АН CCCP, 194:1 (1970), 41-44 (совм. с В. Б. Барановым, К. В. Краснобаевым); англ. пер.: "A model of the interaction of the solar wind with the interstellar medium", Soviet Phys. Dokl., 15:791-793 (1971) (with V. B. Baranov, K. V. Krasnobaev).

[22] “Фронты ионизации и рекомбинации в электромагнитном поле", Итоги науки. Гидромеханика, 5, ВИНИТИ, М., 1971, 5-31 (совм. с А. А. Барминым).

[23] "О течениях проводящей несжимаемой жидкости в произвольной области при наличии сильного магнитного поля", Изв. АН СССР. Сер. механ. жидкости и газа, 8:3 (1973), 144-150; англ. пер.: "Flows of a conducting incompressible liquid in an arbitrary region with a strong magnetic field", Fluid Dynam., 8:3 (1973), 462-467.

[24] "Двумерная задача о движении снежной лавины по склону с плавно меняющимися свойствами", ПММ, 37:5 (1973), 837-848 (совм. с М. Э. Эглит); англ. пер.: "Two-dimensional problem of the motion of a snow avalanche along a slope with smoothly changing properties", J. Appl. Math. Mech., 37:5 (1973), 792-803 (with M. È. Èglit).

[25] "О выборе автомодельного решения в теории пограничного слоя", Изв. АН СССР. Сер. механ. жидкости и газа, 1974, № 4, 42-46 (совм. с Ф. А. Слободкиной); англ. пер.: "Choice of a self-similar solution in boundary-layer theory", Fluid Dynam., 9:4 (1974), 536-539 (with F. A. Slobodkina).

[26] "Задача о поршне при наличии фронтов рекомбинации в магнитном поле", Изв. АН СССР. Сер. механ. жидкости и газа, 10:4 (1975), 136-142 (совм. с А. А. Барминым); англ. пер.: "Piston problem in the presence of recombination waves in a magnetic field", Fluid Dynam., 10:4 (1975), 653-658 (with A. A. Barmin).

[27] "Об устойчивости течений, возникающих при распадении произвольного разрыва", ПММ, 39:1 (1975), 95-102 (совм. с Г. Я. Галиным); англ. пер.: "On the stability of flows appearing at the disintegration of an arbitrary discontinuity", J. Appl. Math. Mech., 39:1 (1975), 85-92 (with G. Ia. Galin).

[28] "О границах области роста неодномерных возмущений неустойчивых состояний", ПММ, 41:6 (1977), 1122-1123 (совм. с В. А. Кондрашевым); англ. пер.: "On boundaries of the growth region of multidimensional perturbations of unstable states", J. Appl. Math. Mech., 41:6 (1977), 1141-1142 (with V. A. Kondrashev).

[29] "О свойствах ударных адиабат в окрестности точек Жуге", Изв. АН СССР. Сер. механ. жидкости и газа, 14:2 (1979), 184-186; англ. пер.: "Properties of shock adiabats in the neighborhood of Jouguet points", Fluid Dynam., 14:2 (1979), 317-320. 
[30] "Об устойчивости одномерных течений газа в расширяющихся областях", Изв. АН СССР. Сер. механ. жидкости и газа, 16:2 (1981), 112-119 (совм. с Г. Я. Галиным); англ. пер.: "Stability of one-dimensional gas flows in expanding regions", Fluid Dynam., 16:2 (1981), 246-252 (with G. Ya. Galin).

[31] "Исследование ударной адиабаты квазипоперечных ударных волн в предварительно напряженной упругой среде", ПММ, 46:5 (1982), 831-840 (совм. с Е.И. Свешниковой); англ. пер.: "Investigation of the shock adiabat of quasitransverse shock waves in a prestressed elastic medium", J. Appl. Math. Mech., 46:5 (1982), 667-673 (with E. I. Sveshnikova).

[32] "Об устойчивости одномерных стационарных решений гиперболических систем дифференциальных уравнений при наличии точек обращения в нуль одной из характеристических скоростей”, ПММ, 48:3 (1984), 414-419 (совм. с Ф. А. Слободкиной); англ. пер.: "On the stability of one-dimensional stationary solutions of hyperbolic systems of differential equations containing points at which one characteristic velocity becomes zero", J. Appl. Math. Mech., 48:3 (1984), 299-302 (with F. A. Slobodkina).

[33] "О возможном влиянии колебаний в структуре разрыва на множество допустимых разрывов", Докл. АН СССР, 275:6 (1984), 1349-1352; англ. пер.: "Тhe possible effect of oscillations in a discontinuity structure on the set of admissible discontinuities", Soviet Phys. Dokl., 29:4 (1984), 283-285.

[34] "О некоторых свойствах ударной адиабаты квазипоперечных упругих волн", ПММ, 48:5 (1984), 793-798 (совм. с Е.И. Свешниковой); англ. пер.: "Some properties of the shock adiabat of quasitransverse elastic waves", J. Appl. Math. Mech., 48:5 (1984), 575-578 (with E. I. Sveshnikova).

[35] "Об условиях устойчивости стационарных состояний или течений в областях, протяженных в одном направлении", ПММ, 49:3 (1985), 411-418; англ. пер.: "On the stability conditions for stationary states or flows in regions extended in one direction", J. Appl. Math. Mech., 49:3 (1985), 316-321.

[36] "Об асимптотическом поведении локализованных возмущений при неустойчивости Кельвина-Гельмгольца", Изв. АН СССР. Сер. механ. жидкости и газа, 20:2 (1985), 23-30 (совм. с И. С. Шикиной); англ. пер.: "On the asymptotic behavior of localized perturbations in the presence of Kelvin-Helmholtz instability", Fluid Dynam., 20:2 (1985), 186-193 (with I. S. Shikina).

[37] "Возникновение периодических режимов в стационарных сверхзвуковых МГД течениях вследствие выключения электропроводности среды", Изв. АН СССР. Сер. механ. жидкости и газа, 20:4 (1985), 138-149 (совм. с А. А. Барминым, А. П. Глиновым); англ. пер.: "Occurrence of periodic regimes in steady supersonic MHD flows due to loss of electrical conductivity of medium", Fluid Dynam., 20:4 (1985), 613-623 (with A. A. Barmin, A. P. Glinov).

[38] "Автомодельная задача о действии внезапной нагрузки на границу упругого полупространства", ПММ, 49:2 (1985), 284-291 (совм. с Е.И. Свешниковой); англ. пер.: "A selfsimilar problem on the action of a sudden load on the boundary of an elastic half-space", J. Appl. Math. Mech., 49:2 (1985), 214-220 (with E. I. Sveshnikova).

[39] "Об уравнениях, описывающих распространение нелинейных квазипоперечных волн в слабоанизотропном упругом теле", ПММ, 50:4 (1986), 597-604; англ. пер.: "Equations describing the propagation of non-linear quasitransverse waves in a weakly non-isotropic elastic body", J. Appl. Math. Mech., 50:4 (1986), 455-461.

[40] "О структуре квазипоперечных упругих ударных волн", ПММ, 51:6 (1987), 926-932 (совм. с Е.И. Свешниковой); англ. пер.: "On the structure of quasitransverse elastic shock waves", J. Appl. Math. Mech., 51:6 (1987), 711-716 (with E. I. Sveshnikova). 
[41] "О распаде произвольного начального разрыва в упругой среде", ПМM, 52:6 (1988), 1007-1012 (совм. с Е.И. Свешниковой); англ. пер.: "Тhe decay of an arbitrary initial discontinuity in an elastic medium", J. Appl. Math. Mech., 52:6 (1988), 786-790 (with E. I. Sveshnikova).

[42] "Особенности поведения нелинейных квазипоперечных волн в упругой среде при малой анизотропии", Современные математические проблемы механики и их приложения, K 80-летию со дня рождения академика Леонида Ивановича Седова, Тр. МИАН СССР, 186, Наука, М., 1989, 132-139; англ. пер.: "Peculiarities of the behavior of nonlinear quasitransversal waves in an elastic medium with a small anisotropy", Proc. Steklov Inst. Math., 186 (1991), 153-160.

[43] "О фронтах сильного и слабого разрыва в решениях уравнений разномодульной теории упругости”, ПММ, 53:2 (1989), 294-300 (совм. с Л. А. Пекуровской); англ. пер.: "On fronts of strong and weak discontinuities in solutions of the equations of different-modulus elasticity theory", J. Appl. Math. Mech., 53:2 (1989), 230-235 (with L. A. Pekurovskaya).

[44] "О продольных волнах в упругой среде с кусочно-линейной зависимостью напряжения от деформации”, ПММ, 54:5 (1990), 807-813 (совм. с Л. А. Пекуровской); англ. пер.: "Longitudinal waves in an elastic medium with a piecewise-linear dependence of the stress on the strain", J. Appl. Math. Mech., 54:5 (1990), 663-668 (with L. A. Pekurovskaya).

[45] "Об устойчивости решений некоторых краевых задач для гиперболических уравнений", ПММ, 56:1 (1992), 40-51 (совм. с С. А. Егорушкиным); англ. пер.: "Тhe stability of the solutions of some boundary-value problems for hyperbolic equations", J. Appl. Math. Mech., 56:1 (1992), 36-45 (with S. A. Yegorushkin).

[46] "Волны Римана в упругой среде при малой анизотропии”, ПММ, 57:3 (1993), 90-101 (совм. с Е.И. Свешниковой); англ. пер.: "Riemann waves in an elastic medium with small anisotropy", J. Appl. Math. Mech., 57:3 (1993), 493-502 (with E. I. Sveshnikova).

[47] "О потере устойчивости слабо неоднородными течениями в протяженных областях. Возникновение поперечных колебаний трубы с текущей в ней жидкостью", ПММ, 57:5 (1993), 93-99; англ. пер.: "On the stability loss of weakly non-uniform flows in extended regions. The formation of transverse oscillations of a tube conveying a fluid", J. Appl. Math. Mech., 57:5 (1993), 851-856.

[48] "О существовании и единственности автомодельных решений при наличии точек Жуге на ударной адиабате”, ПММ, 60:1 (1996), 66-71 (совм. с Е. И. Свешниковой); англ. пер.: "The existence and uniqueness of self-similar solutions involving Jouguet points on the shock adiabatic curve", J. Appl. Math. Mech., 60:1 (1996), 61-65 (with E. I. Sveshnikova).

[49] "Об описании длинных нелинейных волн в каналах", Изв. РАН. Сер. механ. жидкости и газа, 31:5 (1996), 136-145 (совм. с Ю. А. Дроздовой); англ. пер.: "Concerning the description of long nonlinear waves in channels", Fluid Dynam., 31:5 (1996), 739-746 (with Yu. A. Drozdova).

[50] "О переходе к неустойчивости в слабонеоднородных течениях без диссипации", ПММ, 60:3 (1996), 433-437 (совм. с И. С. Шикиной); англ. пер.: "Тhe transition to instability in weakly non-uniform flows without dissipation", J. Appl. Math. Mech., 60:3 (1996), 429-432 (with I. S. Shikina).

[51] "Об электромагнитных волнах и их структуре в анизотропных ферромагнетиках", ПММ, 61:1 (1997), 139-148 (совм. с Н.И. Гвоздовской); англ. пер.: "Electromagnetic shock waves and their structure in anisotropic magnetic materials", J. Appl. Math. Mech., 61:1 (1997), 135-143 (with N. I. Gvozdovskaya).

[52] "Об условиях распада нелинейной волны в вязкоупругой среде", Журн. вычисл. матем. и матем. физ., 38:2 (1998), 315-323 (совм. с А.П. Чугайновой); англ. 
пер.: "Disintegration conditions for a nonlinear wave in a viscoelastic medium", Comput. Math. Math. Phys., 38:2 (1998), 305-312 (with A. P. Chugainova).

[53] "Об устойчивости квазипоперечных ударных волн в анизотропных упругих средах", ПММ, 64:6 (2000), 1020-1026 (совм. с А. П. Чугайновой); англ. пер.: "Тhе stability of quasi-transverse shock waves in anisotropic elastic media", J. Appl. Math. Mech., 64:6 (2000), 981-986 (with A. P. Chugainova).

[54] "Об условиях неустойчивости фронта пламени в слабонеоднородном потоке", Изв. РАН. Сер. механ. жидкости и газа, 35:5 (2000), 12-19 (совм. с И. С. Шикиной); англ. пер.: "Conditions of instability of a flame front in a weakly inhomogeneous flow", Fluid Dynam., 35:5 (2000), 635-641 (with I. S. Shikina).

[55] "Признак несуществования и неединственности решений автомодельных задач механики сплошной среды”, ПММ, 65:6 (2001), 971-982 (совм. с Е. И. Свешниковой); англ. пер.: "А criterion for the non-existence or non-uniqueness of solutions of self-similar problems in the mechanics of a continuous medium", J. Appl. Math. Mech., 65:6 (2001), 941-950 (with E. I. Sveshnikova).

[56] "Об устойчивости к двумерным возмущениям метастабильной ударной волны в вязкоупругой среде", ПММ, 66:1 (2002), 109-117 (совм. с А.П. Чугайновой); англ. пер.: "The stability of a metastable shock wave in a viscoelastic medium under two-dimensional perturbations", J. Appl. Math. Mech., 66:1 (2002), 107-114 (with A. P. Chugainova).

[57] “О фазовых переходах при фильтрации в теплопроводном скелете”, Изв. РАН. Сер. механ. жидкости и газа, 2004, № 3, 85-90; англ. пер.: "Phase transitions in flow through a heat-conducting porous skeleton", Fluid Dynam., 39:3 (2004), 429-434.

[58] "Режимы распространения самоподдерживающихся светодетонационных волн", Изв. РАН. Сер. механ. жидкости и газа, 2005, № 5, 170-181 (совм. с Н. Т. Пащенко); англ. пер.: "Propagation regimes of self-supported light-detonation waves", Fluid Dynam., 40:5 (2005), 818-828 (with N. T. Pashchenko).

[59] "О глобальной неустойчивости однородных течений в неодномерных областях", ПММ, 70:2 (2006), 257-263; англ. пер.: "The global instability of uniform flows in non-one-dimensional regions", J. Appl. Math. Mech., 70:2 (2006), 229-234.

[60] "О развитии возмущений на слабонеоднородном фоне", ПММ, 71:5 (2007), 761-774 (совм. с А. В. Лозовским, Н. Т. Пащенко); англ. пер.: "Evolution of perturbations on a weakly inhomogeneous background", J. Appl. Math. Mech., 71:5 (2007), 690-700 (with A. V. Lozovskii, N. T. Pashchenko).

[61] "Классические и неклассические разрывы в решениях уравнений нелинейной теории упругости", УМН, 63:2(380) (2008), 85-152 (совм. с А. П. Чугайновой); англ. пер.: "Classical and non-classical discontinuities in solutions of equations of non-linear elasticity theory", Russian Math. Surveys, 63:2 (2008), 283-350 (with A. P. Chugainova).

[62] "Автомодельные асимптотики, описывающие нелинейные волны в упругих средах с дисперсией и диссипацией", Ж. вычисл. матем. и матем. физ., 50:12 (2010), 2261-2274 (совм. с А. П. Чугайновой); англ. пер.: "Self-similar asymptotics describing nonlinear waves in elastic media with dispersion and dissipation", Comput. Math. Math. Phys., 50:12 (2010), 2145-2156 (with A. P. Chugainova).

[63] "О стационарной структуре ударных волн в упругих средах и диэлектриках", ЖЭТФ, 137:5 (2010), 973-985 (совм. с А.П. Чугайновой); англ. пер.: "On the steady-state structure of shock waves in elastic media and dielectrics", J. Exp. Theor. Phys., 110:5 (2010), 851-862 (with A. P. Chugainova).

[64] "Влияние малой неоднородности фона на асимптотические свойства линейных возмущений", ПММ, 74:2 (2010), 179-190 (совм. с Н. Т. Пащенко); англ. пер.: "The effect of a small background inhomogeneity on the asymptotic properties of linear perturbations", J. Appl. Math. Mech., 74:2 (2010), 127-134 (with N. T. Pashchenko). 
[65] "Околорезонансные поперечные колебания в упругом слое. Стационарные решения”, ПММ, 75:1 (2011), 27-38 (совм. с Е.И. Свешниковой); англ. пер.: "Near-resonance transverse oscillations in an elastic layer. Steady-state solutions", J. Appl. Math. Mech., 75:1 (2011), 19-26 (with E. I. Sveshnikova).

[66] "О многопараметрических фронтах сильных разрывов в механике сплошных сред", ПММ, 75:4 (2011), 531-550; англ. пер.: "Multi-parameter fronts of strong discontinuities in continuum mechanics", J. Appl. Math. Mech., 75:4 (2011), 378-389.

[67] "Устойчивость фронта пламени в расходящемся потоке", Современные проблемы механики, Сборник статей. K 80-летию со дня рождения академика Андрея Геннадьевича Куликовского, Тр. МИАН, 281, МАИК, М., 2013, 55-67 (совм. c Н. Т. Пащенко); англ. пер.: "Stability of a flame front in a divergent flow", Proc. Steklov Inst. Math., 281:1 (2013), 49-61 (with N. T. Pashchenko).

[68] "Об опрокидывании волн Римана в упругопластических средах с упрочнением", ПММ, 77:4 (2013), 486-500 (совм. с А. П. Чугайновой). 